\title{
Effects of Naltrexone on Large- Scale Network Interactions in Methamphetamine Use Disorder
}

\author{
Milky Kohno ${ }^{1,2,3,4 *}$, Angelica M. Morales ${ }^{1,2}$, Laura E. Dennis ${ }^{1,3}$, Holly McCready ${ }^{1,3}$, \\ William F. Hoffman ${ }^{1,2,3,4,5}$ and P. Todd Korthuis ${ }^{6}$
}

${ }^{1}$ Department of Psychiatry, Oregon Health and Science University, Portland, OR, United States, ${ }^{2}$ Department of Behavioral Neuroscience, Oregon Health and Science University, Portland, OR, United States, ${ }^{3}$ Research and Development Service, Veterans Affairs Portland Health Care System, Portland, OR, United States, ${ }^{4}$ Methamphetamine Abuse Research Center, Oregon Health and Science University and Veterans Affairs Portland Health Care System, Portland, OR, United States, ${ }^{5}$ Mental Health Division, Veterans Affairs Portland Health Care System, Portland, OR, United States, ${ }^{6}$ Section of Addiction Medicine, Oregon Health and Science University, Portland, OR, United States

\section{OPEN ACCESS}

Edited by:

Marc N. Potenza,

Yale University, United States

Reviewed by: Ning Ma,

RIKEN Brain Science Institute (BSI),

Japan

Kesong $\mathrm{Hu}$,

Lake Superior State University,

United States

*Correspondence: Milky Kohno Kohno@ohsu.edu

Specialty section:

This article was submitted to Addictive Disorders,

a section of the journal

Frontiers in Psychiatry

Received: 23 May 2019

Accepted: 30 July 2019 Published: 03 September 2019

Citation:

Kohno M, Morales AM, Dennis LE, McCready H, Hoffman WF and Korthuis PT (2019) Effects of Naltrexone on Large-Scale Network Interactions in Methamphetamine Use Disorder.

Front. Psychiatry 10:603. doi: 10.3389/fpsyt.2019.00603
Naltrexone attenuates craving, and the subjective effects of methamphetamine and extended-release naltrexone (XR-NTX) reduces functional connectivity between regions of the striatum and limbic cortex. Naltrexone modulates neural activity at dopaminergic synapses; however, it is unclear whether naltrexone has an effect on large-scale brain networks. Functional networks interact to coordinate behavior, and as substance-use disorders are associated with an imbalance between reward and cognitive control networks, treatment approaches that target interactive brain systems underlying addiction may be a useful adjunct for behavioral therapies. The objective of this study was to examine the effect of XR-NTX on large-scale brain networks and to determine whether changes in network relationships attenuate drug use, craving, and addiction severity. Thirty-nine participants in or seeking treatment for methamphetamine-use disorder were enrolled in a clinical trial of XR-NTX between May 2013 and March 2015 (Clinicaltrials. gov NCT01822132). Functional magnetic resonance imaging (fMRI) and questionnaires were conducted before and after double-blinded randomization to a 4-week injection of XR-NTX or placebo. In the XR-NTX group, methamphetamine use was reduced along with a decrease in the coupling between executive control (ECN) and default mode (DMN) networks. As decoupling of ECN and DMN networks was associated with change in the severity of dependence, the results suggest that XR-NTX may modulate and enhance ECN attentional resources and suppress DMN self-referential and emotional processing. This study identifies the effect of naltrexone on changes in the intrinsic functional coupling of large-scale brain networks and provides a more systematic understanding of how largescale networks interact to promote behavioral change in methamphetamine-use disorder.

Keywords: naltrexone, resting-state functional magnetic resonance imaging, methamphetamine, striatum, functional connectivity 


\section{INTRODUCTION}

Although methamphetamine (MA) is a highly addictive psychostimulant causing severe physical, neurological, and emotional disruptions (1), there are no FDA-approved medications for MA-use disorder (2). Psychosocial interventions, such as cognitive behavioral therapy, are the mainstay of treatment and are used to strengthen cognitive control over behaviors that promote drug use (2). The efficacy of behavioral interventions for MA use may be undermined by abnormalities in brain structure and function that are associated with impairments in executive functioning $(3,4)$ and linked to clinical features of addiction such as craving (5). Pharmacological interventions that alter neural network connectivity in individuals with MA-use disorders may have the potential to improve treatment outcomes.

Naltrexone, a competitive mu-opioid receptor antagonist, attenuates craving and subjective effects of MA in humans (6, 7). Although a 12-week study showed no differences between extended-release naltrexone (XR-NTX) and placebo on overall MA-use behavior (8), the effects of naltrexone on dopaminergic synapses to strengthen cognitive control could be a useful adjunct to behavioral therapy. Naltrexone, through downstream effects mediated by mu-opioid receptor antagonism, inhibits dopamine signaling in limbic regions. Consistent with naltrexone's pharmacologic actions, we recently demonstrate that XR-NTX decreased connectivity between the nucleus accumbens, amygdala, hippocampus, and midbrain (9) using seed-based resting-state functional connectivity. Studies in healthy controls have demonstrated that changes in dopamine signaling affects the overall topography of resting-state networks that extend beyond dopamine terminal regions $(10,11)$. This study, therefore, examined the impact of XR-NTX on large-scale network interactions that may support behavioral approaches by strengthening cognitive control to abstain from MA use.

Advances in understanding the functional organization of brain systems suggest that a collection of interconnected brain areas work together to form functional networks that interact to coordinate behavior. Core networks include the Default Mode Network (DMN), which is comprised of the posterior cingulate cortex, temporal and medial prefrontal cortices and is associated with self-monitoring function and internal attention; the Executive Control Network (ECN), which includes the dorsolateral prefrontal cortex and posterior parietal cortices and is important for cognitive control; and the Salience Network $(\mathrm{SN})$, which is comprised of the insula, anterior cingulate cortex, amygdala, ventral striatum, dorsomedial thalamus, hypothalamus, and substantia nigra/ventral tegmental area and is responsible for processing motivational stimuli and reward saliency (12). The goal of this study was to determine whether XR-NTX could alter the coupling between the ECN, DMN, and $\mathrm{SN}$ and to assess whether individual differences were associated with reductions in MA use and craving. We anticipated that XR-NTX would increase SN-DMN coupling, and this increase would be associated with reductions in MA craving. Prior work has also demonstrated that individuals with MA use disorder have greater coupling between the ECN and DMN than control participants (13). Effective treatments for MA-use disorders may ameliorate abnormalities in network correlations; therefore, we hypothesize that XR-NTX would decrease coupling between the ECN and DMN.

\section{MATERIALS AND METHODS}

\section{Participants}

Thirty-nine participants were enrolled in a randomized, doubleblind, placebo-controlled clinical trial of XR-NTX (Vivitrol, Alkermes). Participants were recruited from community-based treatment programs and primary care clinics in Portland, Oregon, USA, between May 2013 and March 2015 and were included in a previous study examining seed-based resting-state connectivity (9). Inclusion criteria were a DSM-IV diagnosis for methamphetamine dependence, no other substance dependence except tobacco and/or nicotine dependence, no history of psychiatric disorder except depression and/or posttraumatic stress disorder, aspartate transaminase (AST) and alanine transaminase (ALT) $<5$ times the upper limit of normal, between the ages of 18 and 55 years, right-handed, English speaking, and free of drugs and alcohol $>72 \mathrm{~h}$ and no more than 6 months prior to study assessments. Exclusion criteria included opioid use in the last 30 days or opioid dependence in the past 5 years, asensitivity to naltrexone, PLG (polylactideco-glycolide), carboxymethylcellulose, or any other diluent components, a potential need for opioid analgesics during study period, pregnancy, magnetic resonance imaging (MRI) contraindications, or serious medical illness in the past 30 days.

\section{Study Design}

The study was approved by the Oregon Health and Science University and Veterans Affairs Portland Health Care System Joint Institutional Review Board, and all participants provided written informed consent. At baseline, participants underwent resting-state functional MRI and completed survey assessments (Visit 1). Using a double-blind design, participants were randomized to XR-NTX $(n=19)$ or placebo $(n=20)$ groups based on the output from a computerized random number generator. To reduce issues with study drug adherence challenges associated with daily-dosed oral naltrexone, both the XR-NTX and placebo conditions involved a single 4-week injection, which was donated by the manufacturer. Survey and brain imaging assessments were repeated 4 weeks after baseline scans (Visit 2).

\section{Neuropsychiatric Assessment}

A Mini International Neuropsychiatric Interview (MINI) (14) was conducted to confirm substance dependence diagnoses and psychiatric disorders and the Addiction Severity Index-lite (ASIlite) $(15,16)$ was used to assess past 30 -day substance use. MA craving was measured with a visual analog scale (VAS) ranging from 0 (no craving) to 100 (most intense craving possible) (17). The Substance Dependence Severity Scale (SDSS), which is sensitive to change in clinical status, was administered to assess the severity of substance use (18). 


\section{MRI Imaging Acquisition}

Imaging was performed on a 3 Tesla Siemens TIM Trio MRI scanner. A localizer scan was acquired in order to guide slice alignment during anatomical and functional scans. $\mathrm{A} \mathrm{T}_{2}{ }^{*}$-weighted image was acquired using an echo planar imaging scheme (EPI; 24 slices, $4-\mathrm{mm}$ thick, gap width $=1 \mathrm{~mm}$, TR/TE $/ \alpha=2,000 \mathrm{~ms} / 38$ $\mathrm{ms} / 80^{\circ}$, matrix $=128 \times 128, \mathrm{FOV}=240 \times 240 \mathrm{~mm}, 170$ volumes, in-plane pixel size of $1.9 \mathrm{~mm}^{2}$ ), while subjects stared at a white cross on a black screen for $6 \mathrm{~min}$. One high-resolution $\mathrm{T}_{1}$-weighted magnetically prepared rapid acquisition gradient echo (MPRAGE; 76 slices, $1-\mathrm{mm}$ thick, TR/TE/TI/ $\alpha=2,300 \mathrm{~ms} / 3.4 \mathrm{~ms} / 1,200$ $\mathrm{ms} / 12^{\circ}, \mathrm{FOV}=224 \times 256 \mathrm{~mm}$ ) was acquired for co-registration with functional images and statistical overlay.

\section{Resting-State Processing and Group-Level Analyses} Image analysis was performed using FSL 5.0.2.1 (www.fmrib. ox.ac.uk/fsl). Images were skull-stripped, spatially smoothed [5-mm full width at half maximum (FWHM) Gaussian kernel], and realigned to compensate for motion (19). Automatic Removal of Motion Artifacts (AROMA) was then used to reduce motion-induced signal variation using independent component analysis (ICA) with a classifier that uses two temporal and two spatial features to remove motion artifacts. To identify large-scale resting-state networks across all subjects, cleaned outputs from AROMA underwent ICA analysis with Multivariate Exploratory Linear Optimized Decomposition into Independent Components (MELODIC). The number of components generated was not restricted, and 67 group-average independent components were identified. The spatial maps of these independent components were cross correlated with resting-state templates (20) to identify ECN, DMN, and SN networks (Figure 1). FSL's dual regression was used to regress the group spatial maps of the ECN (left and right), DMN, and SN networks for each subject to identify subject-specific spatial maps of each network. For each subject and scan, average time courses were extracted for ECN, DMN, and SN networks. Time courses from the ECN, DMN, and SN for each scan were imported into $\mathrm{R}$ (version 3.3.2) and used to generate between pairwise Pearson correlations between each network for each subject. Correlation coefficients were converted to $\mathrm{z}$-scores via Fisher's transformation.

\section{Statistical Analysis}

Student's t-tests and Fisher's exact tests, where appropriate, were used to compare groups in baseline demographic and clinical variables (Table 1). Repeated measures ANOVAs were used to examine the effects of XR-NTX on craving and MA use. The main effect of treatment (XR-NTX or Placebo) and time (Visit 1 or Visit 2 ) and the interaction of treatment and time were examined on each measure separately. To assess changes in large-scale restingstate network correlations, two-way repeated measures ANOVAs on pairwise correlations between the ECN, DMN, and SN were tested for main effects of group (XR-NTX and Placebo), time (Scan 1 and Scan 2), and the interaction of group by time in SPSS 22. Bivariate correlations were conducted to explore the association between changes in network coupling associated with XR-NTX administration and changes in craving, frequency of MA use, and substance dependence severity between Visit 1 and Visit2.

\section{Results}

\section{Participant Characteristics}

A total of 220 individuals were screened for the study, and 104 were eligible for participation. The most common reasons for exclusion at pre-screening were polysubstance use, abstinence from MA for over 6 months, and MRI contraindications. Of the 104 eligible participants, 52 were randomized (50\% of those who were eligible; $23.6 \%$ of those screened). Three eligible participants declined randomization. Of those randomized, 39 completed baseline and follow-up assessments that were available for analysis. Reasons for exclusion from analysis of those randomized included scheduling conflicts/no-shows and MRI confounds.

At baseline, groups were well-matched on demographic variables (Table 1). Participants did not differ by mean age

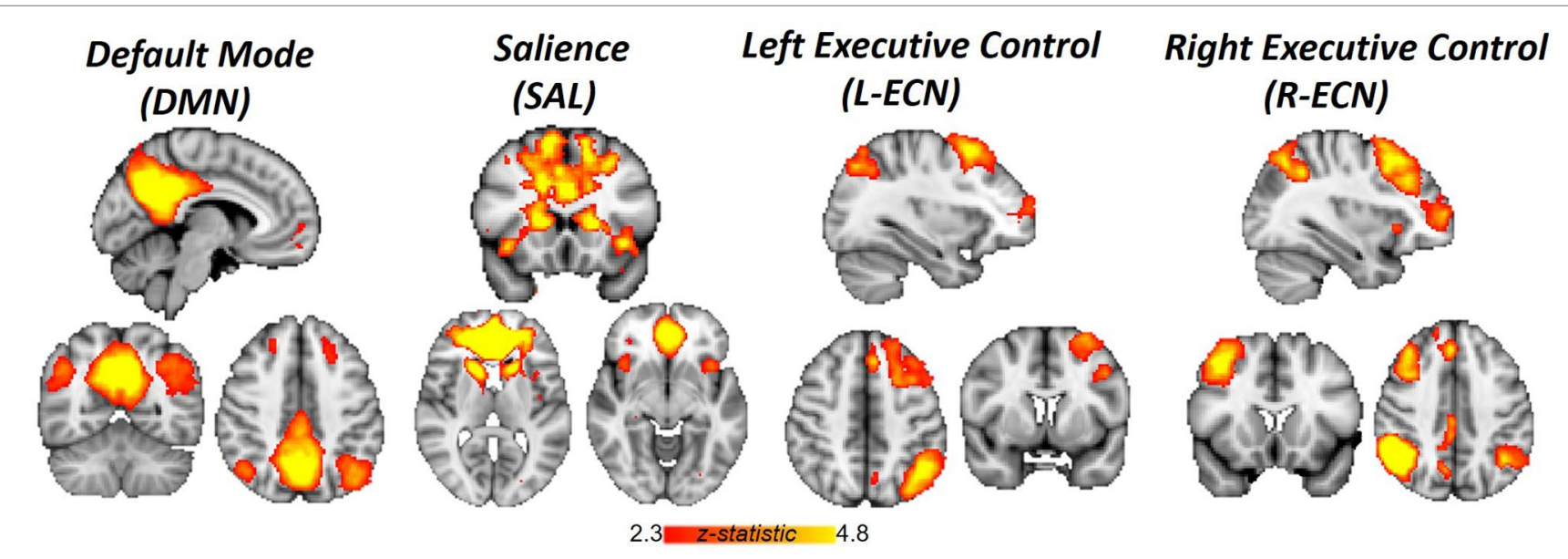

FIGURE 1 | Networks identified by independent component analysis. Spatial maps generated with group ICA and cross-correlated to resting-state template masks include Default Mode Network, Salience Network, and Left and Right Executive Control Network. 
TABLE 1 | Participant Characteristics.

\begin{tabular}{|c|c|c|c|}
\hline & $\begin{array}{c}\text { Placebo } \\
(n=20)\end{array}$ & $\begin{array}{c}\text { NTX } \\
(n=19)\end{array}$ & p-value \\
\hline Age (years) a & $36.47 \pm 10.06$ & $38.68 \pm 9.30$ & 0.49 \\
\hline $\operatorname{Sex}(M / F)^{b}$ & $15 / 5$ & $15 / 4$ & 0.77 \\
\hline Education & $12.63 \pm 0.83$ & $12.78 \pm 2.13$ & 0.78 \\
\hline \multicolumn{4}{|l|}{ Craving } \\
\hline Baseline & $23.84 \pm 27.27$ & $32.83 \pm 27.69$ & 0.33 \\
\hline Follow-up & $18.63 \pm 25.16$ & $20.06 \pm 25.63$ & 0.87 \\
\hline \multicolumn{4}{|c|}{ MA use: Days in the last 30} \\
\hline Baseline & $3.55 \pm 6.42$ & $5.05 \pm 6.93$ & 0.51 \\
\hline Follow-up & $3.87 \pm 7.09$ & $1.56 \pm 3.45$ & 0.23 \\
\hline \multicolumn{4}{|c|}{ Substance Use Severity Scale } \\
\hline Baseline & $5.37 \pm 3.76$ & $8.17 \pm 4.30$ & 0.04 \\
\hline Follow-up & $3.84 \pm 4.25$ & $3.42 \pm 4.23$ & 0.76 \\
\hline Smoking & 17 & 14 & 0.34 \\
\hline \multicolumn{4}{|l|}{ Number of smokers ${ }^{b}$} \\
\hline Positive HIV Status b & 5 & 6 & 0.65 \\
\hline
\end{tabular}

${ }^{a}$ Data shown are means \pm Standard Deviations.

${ }^{b}$ Data analyzed with Chi-squared test $\left(X^{2}\right)$.

(Placebo: 36.47 years; XR-NTX: 38.68 years, $\mathrm{p}=0.49$ ), sex (Placebo: 75\% men; XR-NTX: 79\% men, $\mathrm{p}=0.772$ ), mean years of education (Placebo: 12.63 years; XR-NTX: 12.78 years, $\mathrm{p}=0.78$ ), or smoking status (Placebo: 85\%: XR-NTX: 74\%, $\mathrm{p}=0.34$ ). There were no significant group differences in MA use in the 30 days prior to study enrollment (Placebo: 3.55 days; XR-NTX: 5.05 days, $\mathrm{p}=0.444$ ), craving for MA indexed by the VAS (Placebo: 23.84; XR-NTX: 32.83, $\mathrm{p}=0.327$ ), or HIV status (XR-NTX: 25\%; Placebo group: 32\%, $\mathrm{p}=0.65$ ) but significant differences in SDSS (Placebo: 5.37; XR-NTX: 8.17, $\mathrm{p}=0.04$ ). One HIV-positive subject in each group had no current or past history of taking stable antiretroviral therapy, but all other HIVpositive patients were taking stable antiretroviral therapy prior to and during the study.

\section{Change in Methamphetamine Use, Substance Dependence Severity, and Craving}

On average, the number of days in the past 30 days of selfreported MA use decreased from 5.05 to 1.56 in the XR-NTX group but increased in the Placebo group from 3.55 to 3.87 in the Placebo group. The repeated measures ANOVA resulted in a significant time by treatment interaction $(p=0.03)$, with the XR-NTX group showing greater reductions in MA use compared to the Placebo group. Mean craving scores decreased from 32.83 to 20.07 in the XR-NTX group and from 23.84 to 18.63 in the Placebo group. There were no significant time by treatment interactions on craving and $(\mathrm{p}=0.52)$ or on SDSS $(\mathrm{p}=0.13)$.

\section{Changes in Coupling Between ECN, DMN, and SN}

Correlations between networks for each subject and scan are depicted in Figure 2 for illustrative purposes. In the repeated measures group analyses, a significant interaction of group and time was seen in the correlation between Left ECN and DMN connectivity ( $\mathrm{p}=0.002$, corrected for multiple comparisons) (Figure 3), with no significant differences in correlation at Time $1(p>0.05)$ or at Time $2(p>0.05)$. The group by time interaction remained significant $(\mathrm{p}=0.01)$ after controlling for the group difference in SDSS. There were no significant group by time interactions on the connectivity between SN and Left ECN ( $p=0.086), S N$ and Right ECN ( $p=0.280)$, DMN and SN ( $p=0.704)$, DMN and Right ECN ( $p=0.898)$, or Left ECNRight ECN $(\mathrm{p}=0.424)$.

\section{Relationship between resting-state functional connectivity and clinical outcome measures}

As a group by time interaction on Left ECN and DMN correlations was detected, we explored how changes in Left ECN-DMN coupling affect MA use, craving, and substance dependence severity. When examining the relationship between change in MA use and change in network coupling, we found a significant group interaction (Figure 4, p = 0.04); where the XR-NTX group showed a positive relationship between change in Left ECN-DMN network coupling and change in MA use, while the Placebo group showed a negative relationship. Similarly, the groups differed in the relationship between Left ECN-DMN network change and substance dependence severity (Figure 4, group by connectivity interaction: $\mathrm{p}=0.014$ ), where the XR-NTX group showed a positive relationship and the Placebo group showed a negative relationship. Results remained significant after controlling for duration of MA abstinence $(p=0.003)$, confirming that changes in ECN-DMN coupling was an effect of group and not reductions in MA use. There were no significant interactive effects of group and connectivity on craving ( $p>0.05)$.

\section{DISCUSSION}

This study provides novel evidence indicating that XR-NTX modulates connectivity between large-scale brain networks in individuals with MA dependence. Specifically, XR-NTX reduced coupling between the Left ECN and DMN, which was related to a reduction in MA use and severity of substance dependence. These results are consistent with the role of the ECN in directing attention toward relevant stimuli, flexibly responding to shifting 

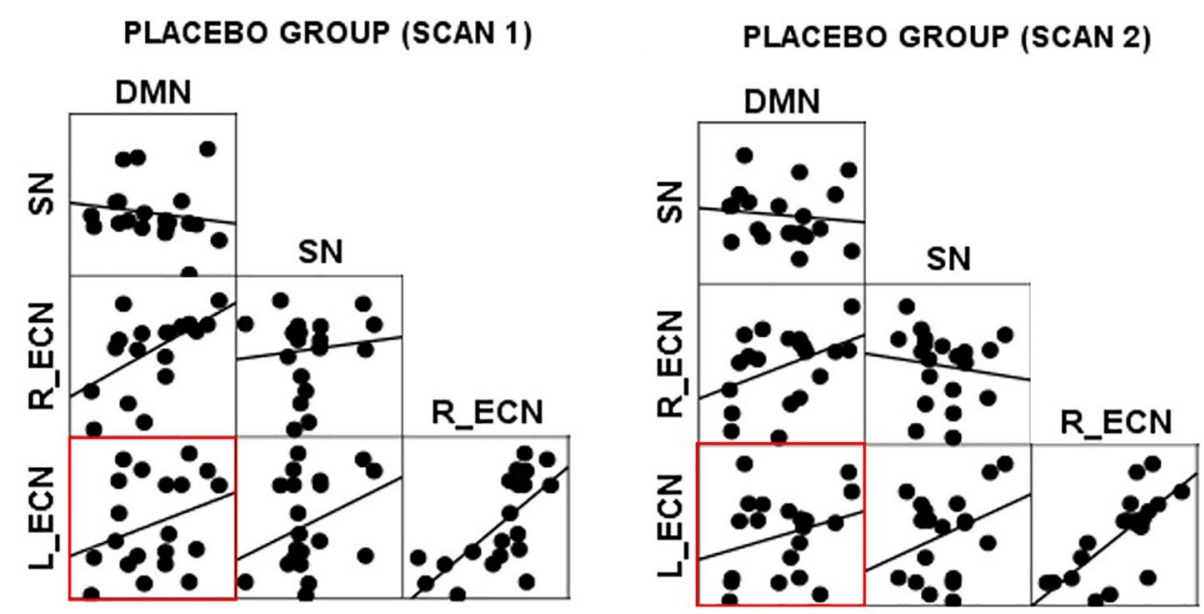

\section{XR-NTX GROUP (SCAN 1)}

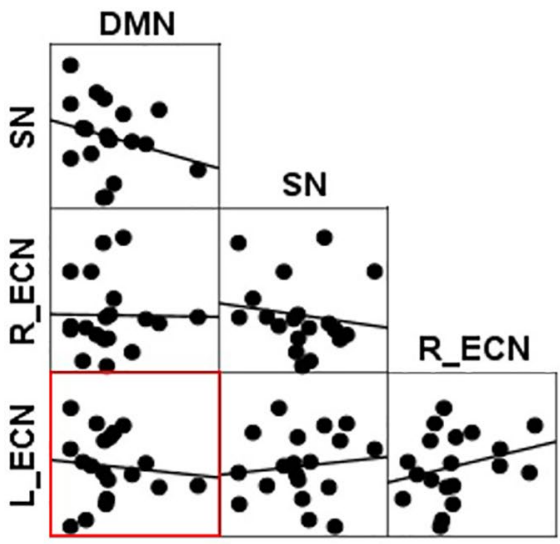

\section{XR-NTX GROUP (SCAN 2)}

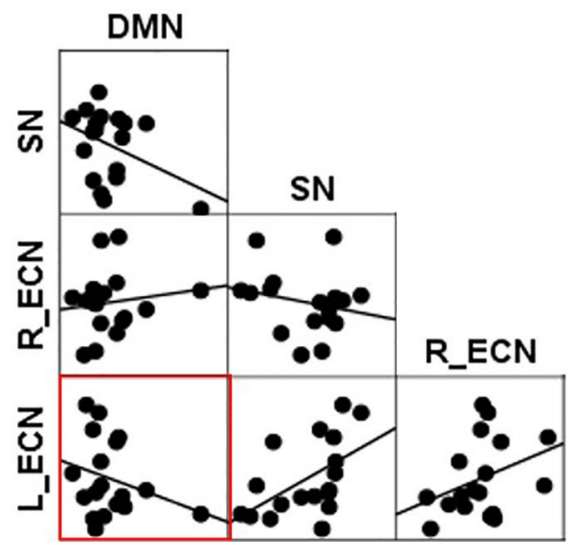

FIGURE 2 | Network correlations. Scatter plots depict the relationships between networks in each group for each scan.

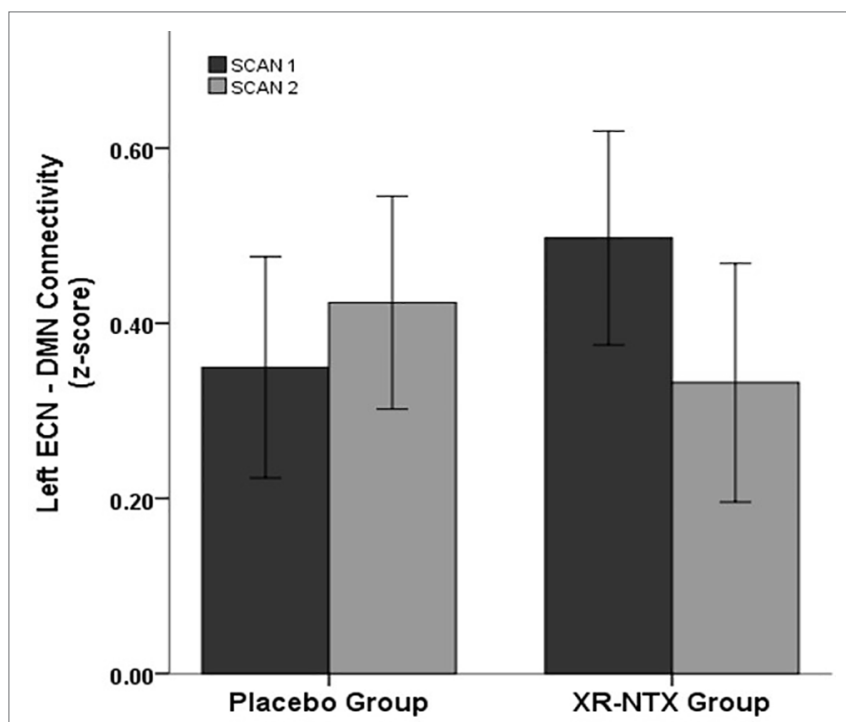

FIGURE 3 | Change in network correlations. Left ECN-DMN correlation. The XR-NTX group show significant reductions between Scan 1 and Scan 2 in Left ECN-DMN coupling compared to the placebo group ( $p=0.002)$. conditions and executing goal-directed behavior (12) and the DMN in processing internal states and episodic memory (21). Given that the activity of ECN and DMN are often anticorrelated and the decoupling from the DMN enables the ECN to allocate attentional resources and to flexibly switch attention in the face of changing cognitive demand, XR-NTX-induced decreases in network coupling may enhance network dynamics to strengthen cognitive resources to limit drug use. In a recent study, baseline DMN connectivity was a predictive factor for treatment outcome in obsessive-compulsive disorder, suggesting that brain connectivity patterns may reflect plasticity of networks that facilitate cognitive and behavioral change (22). As cognitive behavioral therapy requires cognitive flexibility to regulate craving and withdrawal, XR-NTX may be a useful adjunct to treatment to induce network changes that enable plasticity of executive control networks to function without constraint of selfreferential DMN activity during abstinence.

Our findings provide support that medication-induced alterations in dopamine signaling impact resting-state connectivity between the ECN and DMN in individuals with a MA dependence. In particular, one study found that MA-dependent individuals with and without MA-induced 

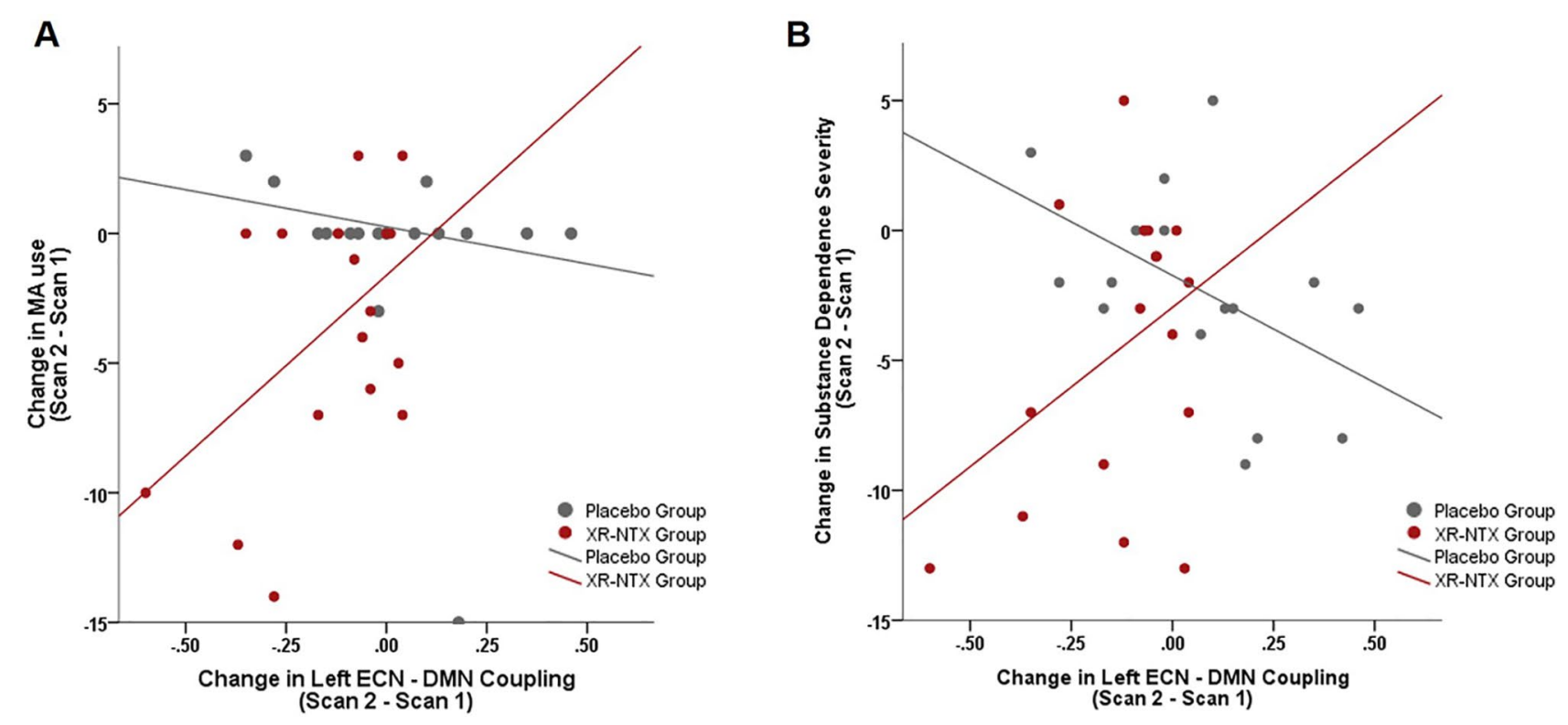

FIGURE 4 | Change in Left ECN-DMN correlations is associated with change in MA use and Substance Dependence Severity. (A) In the XR-NTX group, individuals with greater reductions in network correlations exhibit greater reductions in MA use between Scan 1 and Scan 2 with opposite effects in the Placebo Group ( $p=$ 0.019). (B) In the XR-NTX group, individuals with greater reductions in network correlations exhibit greater reductions in Substance Dependence Severity scores between Scan 1 and Scan 2 with opposite effects in the Placebo Group ( $p=0.014)$.

psychosis had greater ECN-DMN connectivity than control participants (13). In the group of individuals with MA-induced psychosis, lower ECN-DMN connectivity was associated with longer exposure to antipsychotic medications such as the dopamine D2 receptor antagonist, haloperidol. In addition, this study showed that the duration of antipsychotic medication and ECN-DMN connectivity remained significant when controlling for duration of abstinence from MA, suggesting that changes in ECN-DMN connectivity are related to the medication as opposed to reductions in MA use, which is consistent with our findings. Since both antipsychotic medication and naltrexone impact multiple neurotransmitter systems, studies combining restingstate functional connectivity with positron emission tomography to assess neurotransmitter release or receptor density will be useful for uncovering the molecular underpinning of ECNDMN interactions.

The association between XR-NTX-induced changes in ECNDMN coupling, MA use, and substance dependence severity suggest that interventions that successfully alter ECN-DMN connectivity may be especially useful for treating MA-use disorders. In our sample, individuals with smaller changes in ECN-DMN correlations after XR-NTX used MA more frequently, suggesting that for these individuals, identifying other medications that have a greater impact on ECN-DMN coupling may improve treatment outcomes. For example, modafinil has been shown to increase negative coupling between the ECN and DMN and improve cognitive performance in individuals with alcohol-use disorder (23). Although some studies suggest that modafinil is not effective for decreasing MA use (24), these findings may be attributable to heterogeneous medication responses at the individual level.
Studies have shown a positive effect of naltrexone in reducing MA craving $(6,7)$; however, a recent study showed no differences in MA use after 12 weeks of placebo or XR-NTX (8). Mixed results could be attributed to the positive HIV status in the majority of subjects in the latter study, or it is possible that the effects of naltrexone on MA abstinence are time dependent. In the study where MA use did not differ after 12 weeks between the placebo and XR-NTX groups, the XR-NTX group did show a substantial increase in the proportion of participants abstaining from MA in weeks 3, 4, and 5 with no change in the placebo group. As our results show dynamic change in network interactions during this short window, it is possible that this network change may facilitate early MA abstinence. It is unclear whether groups in our study would have converged in MA use or network dynamics after 12 weeks of treatment, but perhaps early network changes coupled with other treatment approaches or cognitive behavioral therapy can help strengthen cognitive control to limit MA use.

Our findings should be interpreted with consideration of the following potential limitations. Although the difference between placebo and XR-NTX on changes in ECN-DMN coupling remained significant after controlling for frequency of MA use, more research is needed to determine whether changes in ECN-DMN coupling precede and causally impact MA use. Furthermore, our sample was relatively small, precluding our ability to examine whether there were sex by treatment interactions on large-scale network dynamics or clinical variables. In addition, future studies could take a data-driven approach to identify spatially constrained regions that drive alterations in large-scale network interactions. Last, although the placebo and XR-NTX groups were matched for cigarette 
use, smoking has been linked to abnormalities in large-scale network dynamics (25). More research is needed to determine how XR-NTX impacts ECN-DMN coupling in individuals with MA-use disorders who do not smoke cigarettes; however, since the vast majority of individuals with MA-use disorder also smoke cigarettes (26), the design of our study may have greater generalizability.

\section{CONCLUSION}

This study provides new evidence of the effect of naltrexone on large-scale brain network dynamics. As the independence of the ECN from other network activity is thought to enable flexible resource allocation during high cognitive demand, the XR-NTXinduced reduction in network coupling between ECN and DMN may facilitate decreases in MA use. Network modifications can facilitate cognitive and behavioral control; however, substanceuse disorders are accompanied by a number of psychosocial factors that need to be addressed to maintain recovery. Although XR-NTX-induced changes in network dynamics can support behavioral changes, it is likely that a combination of approaches that target neural function and cognitive behavioral changes may provide the most therapeutic benefit. This study provides new information on how network changes can affect MA dependence and use. Future studies are required to understand whether XR-NTX-induced brain changes coupled with behavioral therapy would enhance recovery. Conducting clinical trials with cross-over designs to examine the extent to which various medications can impact relevant biomarkers such as ECN-DMN coupling that facilitate behavioral therapy may be useful for tailored treatments that consider an individual's unique pharmacological response.

\section{DATA AVAILABILITY}

The datasets generated for this study are available on request to the corresponding author.

\section{REFERENCES}

1. Scott JC, Woods SP, Matt GE, Meyer RA, Heaton RK, Atkinson JH, et al. Neurocognitive effects of methamphetamine: a critical review and meta-analysis. Neuropsychol Rev (2007) 17(3):275-97. doi: 10.1007/s11065-007-9031-0

2. Rawson RA, McCann MJ, Flammino F, Shoptaw S, Miotto K, Reiber C, et al. A comparison of contingency management and cognitive-behavioral approaches for stimulant-dependent individuals. Addiction (2006) 101(2):267-74. doi: 10.1111/j.1360-0443.2006.01312.x

3. Dean AC, Morales AM, Hellemann G, London ED. Cognitive deficit in methamphetamine users relative to childhood academic performance: link to cortical thickness. Neuropsychopharmacology (2018) 43(8):1745-52. doi: 10.1038/s41386-018-0065-1

4. Kohno M, Morales AM, Ghahremani DG, Hellemann G, London ED. Risky decision making, prefrontal cortex, and mesocorticolimbic functional connectivity in methamphetamine dependence. JAMA Psychiatry (2014) 71(7):812-20. doi: 10.1001/jamapsychiatry.2014.399

5. Morales AM, Kohno M, Robertson CL, Dean AC, Mandelkern MA, London ED. Gray-matter volume, midbrain dopamine D2/D3 receptors and

\section{ETHICS STATEMENT}

The studies involving human participants were reviewed and approved by OHSU IRB. The patients/participants provided their written informed consent to participate in this study.

\section{AUTHOR CONTRIBUTIONS}

PTK and WH designed and implemented the study. LD and HM managed and oversaw the study implementation. MK conducted the analysis and drafted the manuscript. MK and AM contributed to data interpretation. All authors took part in the revision of the manuscript and approved the article.

\section{FUNDING}

The study was funded by the U.S. National Institutes of Health, National Institute on Drug Abuse (R21DA033182, P50DA018165 07, UG1DA015815, T32DA007262, and T32AA007468), National Center for Research Resources (NCRR), a component of the National Institutes of Health (NIH) and NIH Roadmap for Medical Research [1 UL1 RR024140 01 Oregon Clinical and Translational Research Institute (OCTRI)], and Department of Veterans Affairs Clinical Sciences Research and Development Merit Review Program, I0CX001558 (WH) and Development Career Development Award IK2CX001790 (MK), Oregon Health and Science University Collins Medical Trust Award APSYC0249, and Medical Research Foundation of Oregon APSYC0250. The manufacturer, Alkermes, donated extended-release naltrexone and placebo injections for use in this trial.

\section{ACKNOWLEDGEMENTS}

The authors wish to thank Sarann Bielavitz and Doan Ha for assistance with human subjects review. The authors also wish to thank all the study participants for their time and effort.

drug craving in methamphetamine users. Mol Psychiatry (2015) 20(6):76471. doi: $10.1038 / \mathrm{mp} .2015 .47$

6. Roche DJO, Worley MJ, Courtney KE, Bujarski S, London ED, Shoptaw S, et al. Naltrexone moderates the relationship between cue-induced craving and subjective response to methamphetamine in individuals with methamphetamine use disorder. Psychopharmacology (Berl) (2017) 234(13): 1997-2007. doi: 10.1007/s00213-017-4607-8

7. Ray LA, Bujarski S, Courtney KE, Moallem NR, Lunny K, Roche D, et al. The effects of naltrexone on subjective response to methamphetamine in a clinical sample: a double-blind, placebo-controlled laboratory study. Neuropsychopharmacology (2015) 40(10):2347-56. doi: 10.1038/npp.2015.83

8. Coffin PO, Santos GM, Hern J, Vittinghoff E, Santos D, Matheson T, et al. Extended-release naltrexone for methamphetamine dependence among men who have sex with men: a randomized placebo-controlled trial. Addiction (2018) 113(2):268-78. doi: 10.1111/add.13950

9. Kohno M, Dennis LE, McCready H, Schwartz DL, Hoffman WF, Korthuis PT. A preliminary randomized clinical trial of naltrexone reduces striatal resting state functional connectivity in people with methamphetamine use disorder. Drug Alcohol Depend (2018) 192:186-92. doi: 10.1016/j.drugalcdep.2018.07.045 
10. Carbonell F, Nagano-Saito A, Leyton M, Cisek P, Benkelfat C, He Y, et al. Dopamine precursor depletion impairs structure and efficiency of resting state brain functional networks. Neuropharmacology (2014) 84:90-100. doi: 10.1016/j.neuropharm.2013.12.021

11. Shafiei G, Zeighami Y, Clark CA, Coull JT, Nagano-Saito A, Leyton M, et al. Dopamine signaling modulates the stability and integration of intrinsic brain networks. Cereb Cortex (2019) 29(1):397-409. doi: 10.1093/cercor/bhy264

12. Seeley WW, Menon V, Schatzberg AF, Keller J, Glover GH, Kenna H, et al. Dissociable intrinsic connectivity networks for salience processing and executive control. J Neurosci (2007) 27(9):2349-56. doi: 10.1523/ JNEUROSCI.5587-06.2007

13. Ipser JC, Uhlmann A, Taylor P, Harvey BH, Wilson D, Stein DJ. Distinct intrinsic functional brain network abnormalities in methamphetaminedependent patients with and without a history of psychosis. Addict Biol (2018) 23(1):347-58. doi: 10.1111/adb.12478

14. Sheehan DV, Lecrubier Y, Sheehan KH, Amorim P, Janavs J, Weiller E, et al. The Mini-International Neuropsychiatric Interview (M.I.N.I.): the development and validation of a structured diagnostic psychiatric interview for DSM-IV and ICD-10. J Clin Psychiatry (1998) 59 Suppl 20:22-33; quiz 34-57.

15. McLellan AT, Kushner H, Metzger D, Peters R, Smith I, Grissom G, et al. The fifth edition of the Addiction Severity Index. J Subst Abuse Treat (1992) 9(3):199-213. doi: 10.1016/0740-5472(92)90062-S

16. Cacciola JS, Alterman AI, McLellan AT, Lin YT, Lynch KG. Initial evidence for the reliability and validity of a "Lite" version of the Addiction Severity Index. Drug Alcohol Depend (2007) 87(2-3):297-302. doi: 10.1016/j. drugalcdep.2006.09.002

17. Hamilton CM, Strader LC, Pratt JG, Maiese D, Hendershot T, Kwok RK, et al. The PhenX Toolkit: get the most from your measures. Am J Epidemiol (2011) 174(3):253-60. doi: 10.1093/aje/kwr193

18. Gossop M, Darke S, Griffiths P, Hando J, Powis B, Hall W, et al. The Severity of Dependence Scale (SDS): psychometric properties of the SDS in English and Australian samples of heroin, cocaine and amphetamine users. Addiction (1995) 90(5):607-14. doi: 10.1111/j.1360-0443.1995.tb02199.x

19. Jenkinson M, Bannister P, Brady M, Smith S. Improved optimization for the robust and accurate linear registration and motion correction of brain images. NeuroImage (2002) 17(2):825-41. doi: 10.1006/nimg.2002.1132

20. Smith SM, Fox PT, Miller KL, Glahn DC, Fox PM, Mackay CE, et al. Correspondence of the brain's functional architecture during activation and rest. Proc Natl Acad Sci U S A (2009) 106(31):13040-5. doi: 10.1073/ pnas.0905267106

21. Greicius MD, Krasnow B, Reiss AL, Menon V. Functional connectivity in the resting brain: a network analysis of the default mode hypothesis. Proc Natl Acad Sci U S A (2003) 100(1):253-8. doi: 10.1073/pnas.0135058100

22. Reggente N, Moody TD, Morfini F, Sheen C, Rissman J, O’Neill J, et al. Multivariate resting-state functional connectivity predicts response to cognitive behavioral therapy in obsessive-compulsive disorder. Proc Natl Acad Sci U S A (2018) 115(9):2222-27. doi: 10.1073/pnas.1716686115

23. Schmaal L, Joos L, Koeleman M, Veltman DJ, van den Brink W, Goudriaan AE. Effects of modafinil on neural correlates of response inhibition in alcoholdependent patients. Biol Psychiatry (2013) 73(3):211-8. doi: 10.1016/j. biopsych.2012.06.032

24. Anderson AL, Li SH, Biswas K, McSherry F, Holmes T, Iturriaga E, et al. Modafinil for the treatment of methamphetamine dependence. Drug Alcohol Depend (2012) 120(1-3):135-41. doi: 10.1016/j.drugalcdep.2011.07.007

25. Lerman C, Gu H, Loughead J, Ruparel K, Yang Y, Stein EA. Large-scale brain network coupling predicts acute nicotine abstinence effects on craving and cognitive function. JAMA Psychiatry (2014) 71(5):523-30. doi: 10.1001/ jamapsychiatry.2013.4091

26. Weinberger AH, Sofuoglu M. The impact of cigarette smoking on stimulant addiction. Am J Drug Alcohol Abuse (2009) 35(1):12-7. doi: 10.1080/00952990802326280.

Disclaimer: The contents of this paper do not represent the views of the U.S. Department of Veterans Affairs or the United States Government.

Conflict of Interest Statement: The authors declare that the research was conducted in the absence of any commercial or financial relationships that could be construed as a potential conflict of interest.

Copyright (c) 2019 Kohno, Morales, Dennis, McCready, Hoffman and Korthuis. This is an open-access article distributed under the terms of the Creative Commons Attribution License (CC BY). The use, distribution or reproduction in other forums is permitted, provided the original author(s) and the copyright owner(s) are credited and that the original publication in this journal is cited, in accordance with accepted academic practice. No use, distribution or reproduction is permitted which does not comply with these terms. 